\title{
Growth and shape of indium islands on molybdenum at micro- roughened spots created by femtosecond laser pulses
}

\author{
Authors \\ F. Ringleb ${ }^{a}{ }^{*}$, K. Eylers ${ }^{a}$, Th. Teubner ${ }^{a}$, H.-P. Schramm ${ }^{a}$, C. Symietz ${ }^{b}$, J. Bonse ${ }^{b}$, S. Andree ${ }^{b}$, \\ B. Heidmann ${ }^{c, d}$, M. Schmid, ${ }^{c, d}$, J. Krüger ${ }^{b}$, T. Boeck ${ }^{a}$ \\ a Leibniz-Institute for Crystal Growth, Max-Born-Straße 2, 12489 Berlin, Germany \\ ${ }^{b}$ Bundesanstalt für Materialforschung und -prüfung (BAM), Unter den Eichen 87, 12205 Berlin, Germany \\ 'Department of Physics, Freie Universität Berlin (FUB), Arnimalle 14, 14195 Berlin, Germany \\ ${ }^{d}$ Nanooptische Konzeptr für die PV, Helmholtz Zentrum Berlin (HZB), Hahn-Meitner-Platz 1, 14109 Berlin, Germany
}

\begin{abstract}
Indium islands on molybdenum coated glass can be grown in ordered arrays by surface structuring using a femtosecond laser. The effect of varying the molybdenum coated glass substrate temperature and the indium deposition rate on island areal density, volume and geometry is investigated and evaluated in a physical vapor deposition (PVD) process. The joined impact of growth conditions and spacing of the femtosecond laser structured spots on the arrangement and morphology of indium islands is demonstrated. The results yield a deeper understanding of the island growth and its precise adjustment to industrial requirements, which is indispensable for a technological application of such structures at a high throughput, for instance as precursors for the preparation of $\mathrm{Cu}(\mathrm{In}, \mathrm{Ga}) \mathrm{Se}_{2}$ micro concentrator solar cells.
\end{abstract}

\section{Introduction}

Indium is a highly demanded material for optoelectronic applications, such as for displays, photodiode detectors and solar cells. It is a constituent of $\mathrm{Cu}(\mathrm{In}, \mathrm{Ga}) \mathrm{Se}_{2}$ (CIGSe), which is an excellent absorber material for highly-efficient thin film solar cells [1]. To further enhance the efficiency of CIGSe solar cells and to save expensive raw materials, a new device concept of CIGSe micro concentrator solar cells has been proposed. When a solar cell operates under concentrated illumination, its efficiency can be enhanced since the voltage output increases logarithmically with the concentration factor [2]. Compared to the well-established macroscopic concentrator photovoltaic devices, these micro cells allow a better heat dissipation and thus reduced thermal losses as well as a more compact module design. In the case of CIGSe, proof-of-concept studies have already demonstrated the potential of this approach [3,4,5]. A technologically applicable bottom-up process, however, is missing so far.

In a previous work, we have proposed a bottom-up approach for the preparation of CulnSe $\mathrm{Cr}_{2}$ $\mathrm{Cu}(\mathrm{In}, \mathrm{Ga}) \mathrm{Se}_{2}$ micro absorber arrays from indium island precursors [6]. Such precursors can be grown on a molybdenum film, which is the standard back contact of CIGSe solar cells [7]. Island growth in ordered arrays was achieved by the creation of preferred nucleation sites for indium by substrate patterning using a femtosecond ( $f s$ ) laser along with PVD. In this approach, the fs laser beam is focused at the sample surface, which induces surface modifications of diameters in the desired range. The treatment was applied in the regime of moderate ablation [8], where material removal 
leads merely to a localized surface roughening of the glass substrate. After additional deposition of a thin molybdenum film, the laser-generated surface roughness facilitates the nucleation and growth of islands during subsequent PVD of indium. This scenario is schematically shown in the upper part of Fig. 1, both for island growth on non-patterned (left column) and fs laser patterned substrates (right column). In the lower part of Fig. 1 the successful site-selective control of indium island growth upon fs-laser structuring is clearly seen when comparing the two top-view optical micrographs taken in brightfield mode at the end of the two different processing chains.

For such indium islands to be suitable precursors for absorber islands, they have to meet several requirements. For an optimum use of material, they should exhibit a low height-to-width aspect ratio. Moreover, it is important to ensure a reliable but also exclusive growth of islands at the predefined spots. To accomplish this, the intrinsic island areal density on non-patterned substrates and the spacing of the substrate patterning have to be adjusted to each other. In the case of micro concentrator cells, the ratio between the absorber island spacing and the absorber island diameter determines the concentration and material saving factors. These issues illustrate that it is mandatory to understand the indium island growth mechanisms and to identify the optimum processing conditions for a reliable up-scaling to industrial demands. In this work, we present a detailed study on the influence of the indium deposition rate and substrate temperature on island size, areal density and aspect ratio. In addition, the effect of the fs-laser ablation spot spacing on the island morphology is shown. Since they open up additional opportunities for process optimization, these experiments are a prerequisite for the adjustment of the growth parameters of indium islands to realize the desired array dimensions.

\section{Experimental}

Commercial $50 \times 50 \times 2 \mathrm{~mm}^{3}$ soda-lime floatglass samples (Weidner Glas) served as substrates. Sequential metal deposition was carried out by PVD in a high vacuum chamber with a rear and front heater (for details, refer to Ref. [6]). Deposition of both, molybdenum and indium was carried out at a substrate temperature of $510^{\circ} \mathrm{C}$. Molybdenum films of $320-420 \mathrm{~nm}$ thickness were deposited at rates between 1 and $1.8 \AA / \mathrm{s}$. The layer thickness of indium, which was in the range of $90-110 \mathrm{~nm}$, has merely a nominal meaning due to the formation of islands during deposition.

Laser-based substrate patterning was carried out at ambient atmosphere by a pulsed Ti:sapphire fslaser (Femtolasers, Compact Pro) with a center wavelength of $790 \mathrm{~nm}$, a pulse duration of $30 \mathrm{fs}$, and a maximum repetition rate of $1 \mathrm{kHz}$. The laser beam was directly focused to the substrate by a spherical mirror, resulting in a Gaussian beam profile with a radius $w_{0} \sim 100-130 \mu \mathrm{m}$ at the surface. 20 pulses were applied at each spot with an energy of $340 \mu \mathrm{J}$ and a fluence of $2.8 \mathrm{~J} / \mathrm{cm}^{2}$. Since only parts of the substrate were laser-treated, the island growth on arrayed regions could be directly compared to island growth in untreated areas within the same experiment.

Optical microscopy was carried out in brightfield mode with a Reichert Polyvar 2 microscope equipped with a CCD camera (Nikon DS-5M), a DS-U1 interface and NIS-Elements $F$ imaging software (vers. 3.22). Statistical analysis of the areal distribution of indium islands was realized using the image analysis software imageJ (vers. 1.49) with a self-written script for automated image processing. Nearest neighbor distances (NND) were determined by the calculation of the Euclidean distances of the center points of the islands, i.e. the average values of the $x$-and $y$-coordinates of all pixels belonging to an individual island. Scanning electron microscopic (SEM) investigations were carried out with a Nova 600 Nanolab DualBeam microscope from FEI. A Dimension Icon atomic force microscope (AFM) from Bruker, operated in tapping mode, provided data for the surface roughness of Mo films and the shape of small islands. For these small islands, cross-sectional scans were 
extracted from the AFM images. A Dektak XT stylus profiler (Bruker) was applied for cross-sectional scans of larger islands.

\section{Results and Discussion}

\subsection{Island nucleation without fs laser patterning}

Morphology and distribution of the indium islands both are sensitive to temperature, deposition rate, material and surface structure of the substrate. In the present study, we characterized our molybdenum coated glass substrates by AFM topographical maps and SEM cross-sectional images (data now shown). Thereby, we identified a polycrystalline columnar structure of the Mo film with column axes being oriented normal to the surface, which is typical for molybdenum thin films on soda lime glass used as back contact for $\mathrm{Cu}(\mathrm{In}, \mathrm{Ga}) \mathrm{Se}_{2}$ solar cells [9]. The grain sizes of our films are in the range of $3 \mathrm{~nm}$ and the film surface exhibits a roughness $R_{\mathrm{a}}$ of about $3 \mathrm{~nm}$.

The influence of deposition rate $(r)$ and substrate temperature $(T)$ on the size, distribution and morphology of indium islands is shown in Fig. 2. Each column summarizes the experimental results for a particular combination of $T$ and $r$. For all experiments, $420 \mathrm{~nm}$ thick films of molybdenum and nominal $100 \mathrm{~nm}$ of indium were deposited at standard conditions (see experimental section). Average diameters and nearest neighbor distances (NND) were derived from a statistical analysis (for details, refer to Ref. [6]). Comparing the grain sizes of the molybdenum film ( $\sim 3 \mathrm{~nm}$ ) and the NNDs of the indium islands, it becomes evident that the island spacing is orders of magnitudes larger than the individual molybdenum grains on all samples shown. The islands aspect ratio (height : width) was derived from cross-sectional scans as depicted at the bottom of each column. Due to the large differences of the indium island dimensions, such scans were obtained by AFM for Figs. 2(a) and 2(b) and by profilometric scans for Figs. 2(c), 2(d) and 2(e).

In a first set of experiments, the indium deposition was carried out at different substrate temperatures $\left(T=410^{\circ} \mathrm{C}, 460^{\circ} \mathrm{C}\right.$, and $510^{\circ} \mathrm{C}$, respectively) at a constant indium deposition rate of $r=$ $0.3 \AA$ A $/ \mathrm{s}$ (Fig. 2, columns (a), (b), and (c)). The data shows a trend of increasing island diameters and NNDs with rising temperatures.

This can be intuitively understood considering the higher mobility of indium adatoms at larger $T$. The diffusion coefficient, which rises with increasing temperatures, determines how far an adatom migrates in a certain period of time. Therefore, the probability for an adatom to reach an existing island before meeting another atom, which is arriving from the gas phase, rises with increasing sample temperatures. Furthermore, the nucleation barrier and critical nucleus size increase with temperature [10]. Many experimental studies $[11,12]$ report such a tendency of island density vs. temperature. Monte Carlo simulations [13] support these findings theoretically.

The columns (a)-(c) of Fig. 2 also illustrate that the aspect ratio of the islands decreases at higher substrate temperature. This is presumably a thermodynamic effect related to the temperature dependence of interfacial energies. Theoretical and experimental studies both agree that the surface tension of liquid indium is reduced with increasing temperature [14]. A flattening with increasing island size due to gravitation can be excluded, since the sample surface was facing downwards during evaporation in this study.

In a second set of experiments, the effect of indium deposition rate at a constant substrate temperature was studied, as illustrated in columns (c), (d) and (e) of Fig. 2. Here, indium deposition rates of $r=0.3 \AA \mathrm{s}, 0.5 \AA \mathrm{s}$ and $0.9 \AA \mathrm{A} / \mathrm{s}$ have been applied in combination with a constant substrate temperature of $510^{\circ} \mathrm{C}$. The comparison demonstrates that decreasing indium deposition rates lead to 
similar tendencies as increased substrate temperatures with respect to island diameters and NNDs, i.e. larger island diameters and lower island areal densities. This does not hold, however, for the aspect ratio, which is rather constant between 0.04 and 0.06 for a variation of the deposition rate by a factor of three. This is in clear contrast to the variation of $T$, where the height-to-width ratio changes by approximately one order of magnitude within the investigated temperature range of $100 \mathrm{~K}$. These observations are in line with a surface energy-controlled shape formation. The larger island density and smaller island size at constant temperature (adatom mobility) are in accordance with the relatively simple random walk model of diffusing adatoms. If the flux of impinging atoms rises at a constant temperature, the probability for an adatom to reach an existing island decreases, whereas the nucleation of an additional island becomes more favorable. From a thermodynamic point of view, the nucleation barrier and critical nucleus size decrease with increasing flux, which results in higher nucleation rates [10].

\subsection{Island nucleation with fs laser patterning}

For the arrangement of indium islands in regular arrays, the interplay between growth parameters and substrate patterning has to be taken into account. For obtaining islands of desired morphology, which grow exclusively at the desired laser-treated locations, an adjustment between the intrinsic island areal density (depending on the deposition conditions) and the spacing of the laser ablation spots is indispensable. If the spacing of neighboring laser ablation spots is significantly higher than the NND of indium islands on non-patterned substrate regions, interstitial islands will start to grow.

Fig. 3 presents the results for laser spot spacings roughly in the range of and lower than the intrinsic NND. The arrays consisting of $5 \times 5$ ablation spots with 200, 400, 600 and $800 \mu \mathrm{m}$ spacing shown in this figure were all located on the same sample and exposed to identical experimental conditions. After laser irradiation, a standard molybdenum film was deposited on the glass substrate (see experimental part). Subsequently, indium islands were grown at a rate of $0.3 \AA \mathrm{s}$ at a substrate temperature of $510^{\circ} \mathrm{C}$ (total nominal thickness: $100 \mathrm{~nm}$ ), which yielded a NND of the islands outside of the laser-patterned areas of $750 \mu \mathrm{m}$. Even though the largest array spacing applied here $(800 \mu \mathrm{m})$ is about $7 \%$ higher than this intrinsic NND $(750 \mu \mathrm{m})$, interstitial island growth was suppressed in this case.

On the left-hand side of Fig. 3, the indium islands of each array are all depicted in collages of SEM micrographs recorded at a $52^{\circ}$ tilt with respect to the surface normal. These collages illustrate that, regardless of how much smaller the array spacing is with respect to the intrinsic NND, indium nucleates at each laser-roughened spot. The non-occupation of position $(2,4)$ in the $600 \mu \mathrm{m}$ spaced array is most likely due to laser instabilities, since basically no surface roughening is observed at this position. The reliability of the deposition of indium in the laser ablation spots is of enormous advantage for technological applications.

Another interesting aspect is that the smaller the spacing becomes, the more evident is the material competition. This is reflected by an inhomogeneous distribution of indium island volumes across the array and particularly a reduction of the indium island size in the center. From profilometric crosssectional scans, height and width of each island were determined. Based on this data, the island volumes were estimated using a spherical cap approximation. The calculated volumes are plotted for each array spacing on the right-hand side of Fig. 3., whereby darker gray tones represent larger island volumes. In this data representation, it becomes evident that the smallest islands are located in the center of the arrays. At the array edges, intermediate volumes are observed and the largest 
islands are located at the corners. These tendencies are stronger the smaller the array spacing is chosen.

Fig. 4 shows profilometric cross sections of individual indium islands from each array of Fig. 3 - all from equivalent array positions (fourth line, third column). In addition, the cross section of a bare laser spot (without indium deposition) is provided. Clearly, the substrate surface area covered by the indium islands (and therefore the island volume) decreases with decreasing array spacing. One can approximate, that each island acquires indium from within a "capture zone", which has an area roughly corresponding to the reciprocal island's areal density. In the arrays shown in Fig. 3, the island areal density increases from the $800 \mu \mathrm{m}$ to the $200 \mu \mathrm{m}$ spacing. Therefore, the capture zone, from which the material is acquired gets smaller, which results in smaller island volumes.

The depicted indium islands from the arrays of $400 \mu \mathrm{m}, 600 \mu \mathrm{m}$ and $800 \mu \mathrm{m}$ spacing all exhibit a contact angle $\theta$ of about $10^{\circ}$ (note the different scaling of $x$ and $y$ axes) and only slight variations of the shape with height-to-width aspect ratios of $0.03,0.05$ and 0.05 , respectively. The aspect ratio of the island from the $200 \mu \mathrm{m}$-spaced array, however, is one order of magnitude smaller $(0.008)$. Comparing the indium islands from the $400 \mu \mathrm{m}$ and the $200 \mu \mathrm{m}$-spaced arrays, it is evident that both island diameters are matching with the extent of the laser ablation spot. However, since the capture zone for each island in the $200 \mu \mathrm{m}$ array is smaller than in the $400 \mu \mathrm{m}$ array, the corresponding island volume is smaller in this case. However, indium still wets the entire region, which has been roughened by the fs-laser beam. As a consequence, the aspect ratio is decreased. In summary, the height-to-width ratio can be influenced not only by the substrate temperature during deposition (see Fig. 2), but also by the ratio between laser ablation spot size and deposited volume of indium. This is an interesting point, since flat indium precursor islands with a height $\leq 2 \mu \mathrm{m}$ are desirable for the further processing to micro absorbers for photovoltaics.

\section{Conclusions}

At elevated temperatures, indium shows a strong tendency to dewet on molybdenum substrates and to form three-dimensional islands with a temperature and rate dependent morphology. For the temperature range investigated in this study $\left(410^{\circ} \mathrm{C}-510^{\circ} \mathrm{C} ; 0.3-0.9 \AA / \mathrm{s}\right)$ it was found that: (1) The substrate temperature significantly affects the indium island's density. The temperature-dependent nucleation rate can be explained by a higher mobility of indium atoms at elevated temperatures. Moreover, the island's geometrical aspect ratios change by about one order of magnitude within a temperature range of $100 \mathrm{~K}$, which might be determined by the temperature-dependence of the interfacial energies. (2) The indium deposition rate also affects island density and size. The aspect ratio, however, is almost independent of a variation of the deposition rate by a factor of three. This constitutes a further indication that thermodynamics has a stronger influence on the island shape than kinetics under the applied growth conditions.

Intrinsic island growth and laser patterning have to be adjusted to each other. If the laser spot spacing gets smaller than the intrinsic NND, material competition becomes important. This results in deviations of the island size and shape between the center, edges and corners of the laser spot arrays. For each array spacing investigated here, the island growth at the laser spots was very reliable. If the spot diameter is smaller than the size of the on-top grown island, the island shape corresponds to the shape of freely grown islands, i.e. contact angle and height-to-width aspect ratio remain approximately constant. For islands with diameters of the laser roughened spots, however, a regime can be realized where the aspect ratio can be scaled down, leading to desirable indium island heights $\leq 2 \mu \mathrm{m}$. 


\section{Acknowledgements}

The authors gratefully acknowledge financial support by the Deutsche Forschungsgemeinschaft (DFG) through BO 1129/6-1, KR 3638/3-1, and SCHM 2554/3-1. The research leading to these results has received funding from the European Union Seventh Framework Programme (FP7/2007-2013) under grant agreement $n^{\circ} 609788$.

\section{References}

[1] M. A. Green, K. Emery, Y. Hishikawa, W. Warta, E. D. Dunlop, Solar cell efficiency tables (version 47), Prog. Photovolt Res. Appl. 24 (2015) 3-11.

[2] C. Algora, I. Rey-Stolle, Handbook on Concentrator Photovoltaic Technology, Wiley, 2016

[3] B. Reinhold, M. Schmid, D. Greiner, M. Schüle, D. Kieven, A. Ennaoui, M. C. Lux-Steiner. Monolithically interconnected lamellar $\mathrm{Cu}(\mathrm{In}, \mathrm{Ga}) \mathrm{Se} 2$ micro solar cells under full white light concentration, Prog. Photovoltaics Res. Appl. 23 (2015) 1929-1939.

[4] M. Paire, L. Lombez, F. Donsanti, M. Jubault, S. Collin, J. L. Pelouard, J. F. Guillemoles, D. Lincot, $\mathrm{Cu}(\mathrm{In}, \mathrm{Ga}) \mathrm{Se} 2$ microcells: High efficiency and low material consumption, J. Renew. Sustain. Energy 5 (2013) 1-6.

[5] M. Paire, A. Shams, L. Lombez, N. Péré-Laperne, S. Collin, J.-L. Pelouard, J.-F. Guillemoles, D. Lincot, Resistive and thermal scale effects for $\mathrm{Cu}(\mathrm{In}, \mathrm{Ga}) \mathrm{Se} 2$ polycrystalline thin film microcells under concentration, Energy Environ. Sci. 4 (2011) 4972.

[6] F. Ringleb, K. Eylers, T. Teubner, T. Boeck, C. Symietz, J. Bonse, S. Andree, J. Krüger, B. Heidmann, M. Schmid, M. Lux-Steiner, Regularly arranged indium islands on glass/molybdenum substrates upon femtosecond laser and physical vapor deposition processing, Appl. Phys. Lett. 108 (2016) 111904.

[7] P. C. Huang, C. H. Huang, M. Y. Lin, C. Y. Chou, C. Y. Hsu, C. G. Kuo, The effect of sputtering parameters on the film properties of molybdenum back contact for CIGS solar cells, Int. J. Photoenergy 2013 (2013).

[8] D. Bäuerle, Laser Processing and Chemistry, fourth ed., Springer, 2011

[9] P. M. P. Salomé, J. Malaquias, P. A. Fernandes, A. F. Da Cunha, Mo bilayer for thin film photovoltaics revisited, J. Phys. D Appl. Phys. 43 (2010) 345501-7.

[10] M. Ohring, Materials Science of Thin Films, second ed., Academic Press, 2001.

[11] H. J. Kim, Z. M. Zhao, J. Liu, V. Ozolins, J. Y. Chang, Y. H. Xie, A technique for the measurement of surface diffusion coefficient and activation energy of Ge adatom on Si(001), J. Appl. Phys. 95 (2004) 6065-6071. 
[12] J. H. Lee, Z. M. Wang, G. J. Salamo, Observation of change in critical thickness of In droplet formation on $\operatorname{GaAs}(100)$, J. Phys. Condens. Matter 19 (2007) 176223.

[13] M. C. Bartelt, J. W. Evans, Scaling analysis of diffusion-mediated island growth in surface adsorption processes, Phys. Rev. B 46 (1992) 12675-12687.

[14] B. B. Alchagirov, R. K. Dadashev, F. F. Dyshekova, D. Z. Elimkhanov, The surface tension of indium: Methods and results of investigations, High Temp. 52 (2014) 920-938. 


\section{glass}

molybdenum

indium

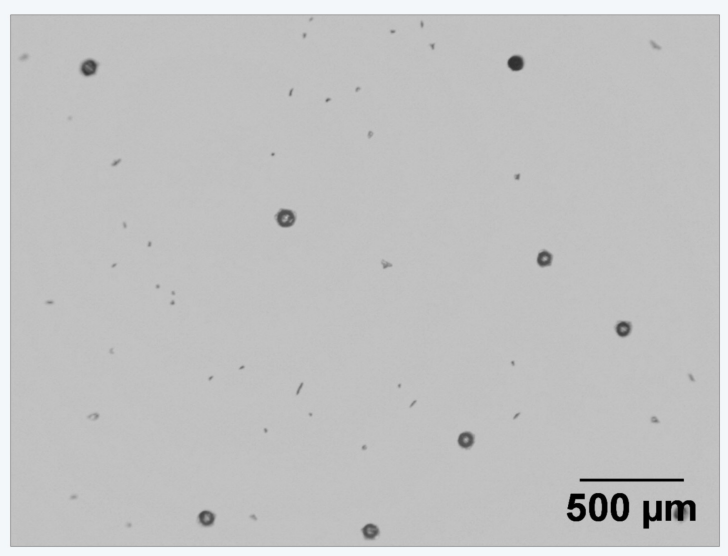

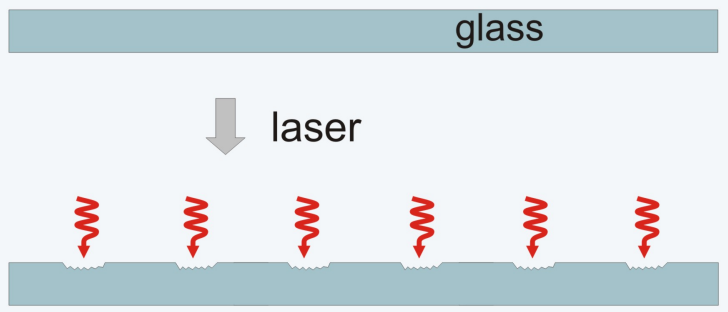

molybdenum

indium

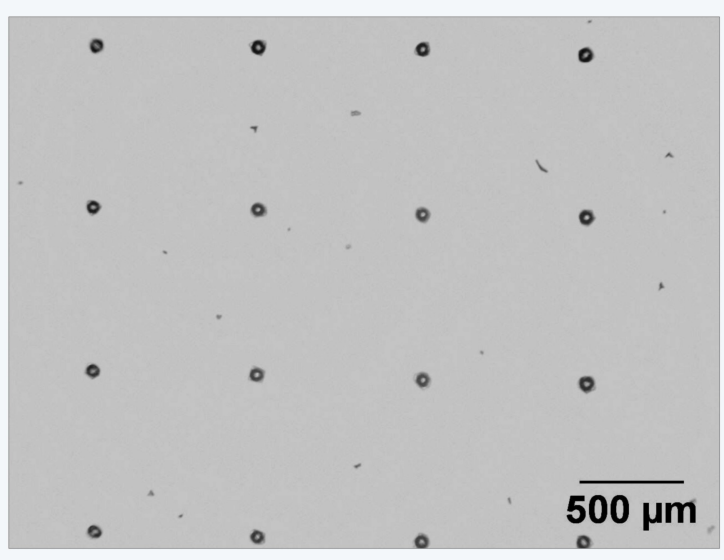


(a)

(b)

(c)

(d)

(e)
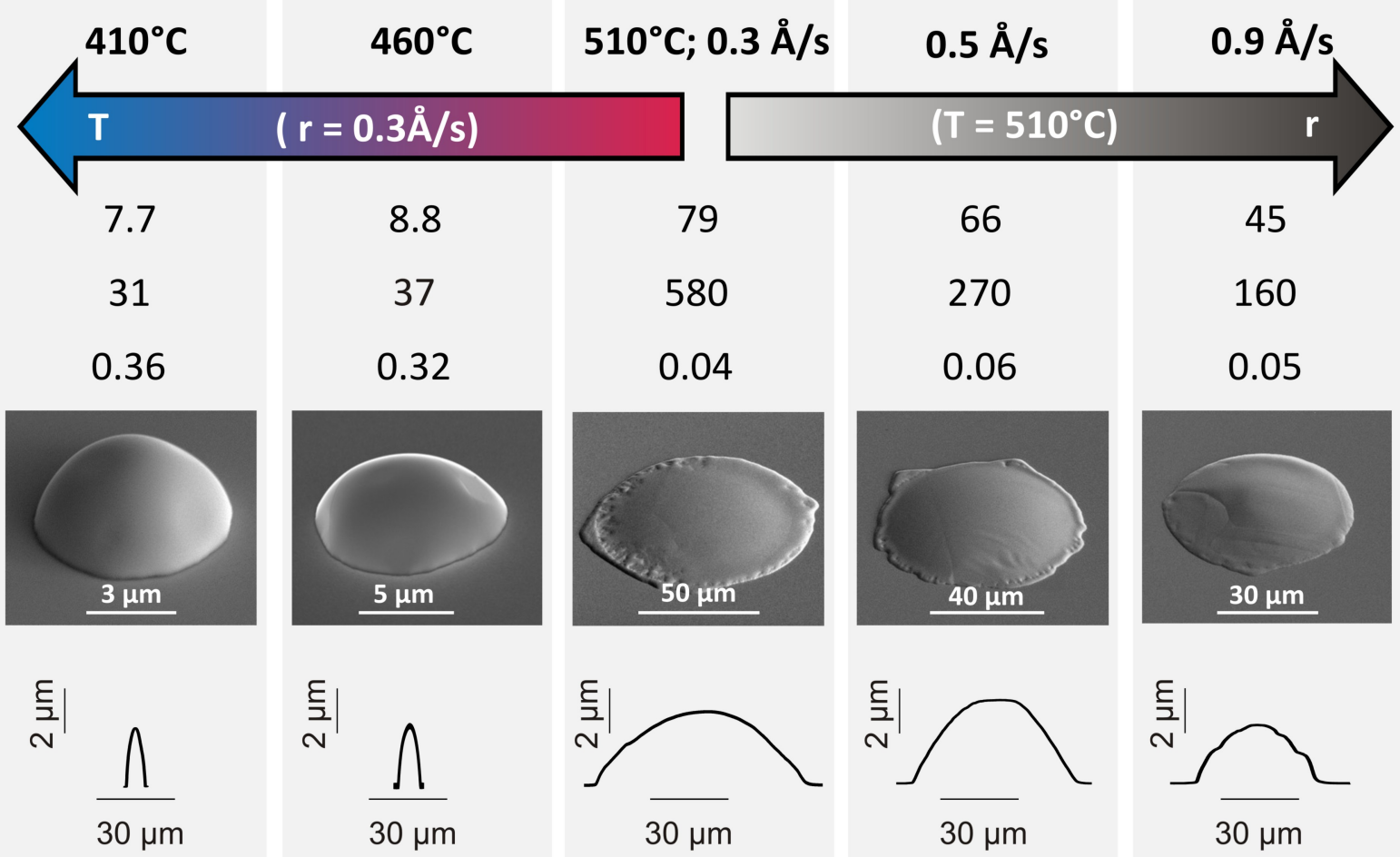
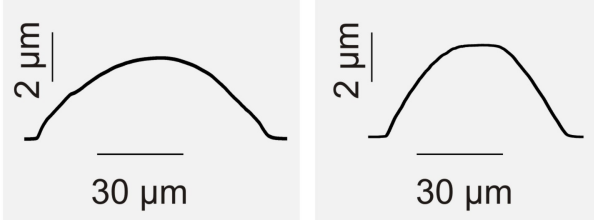

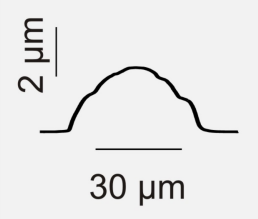



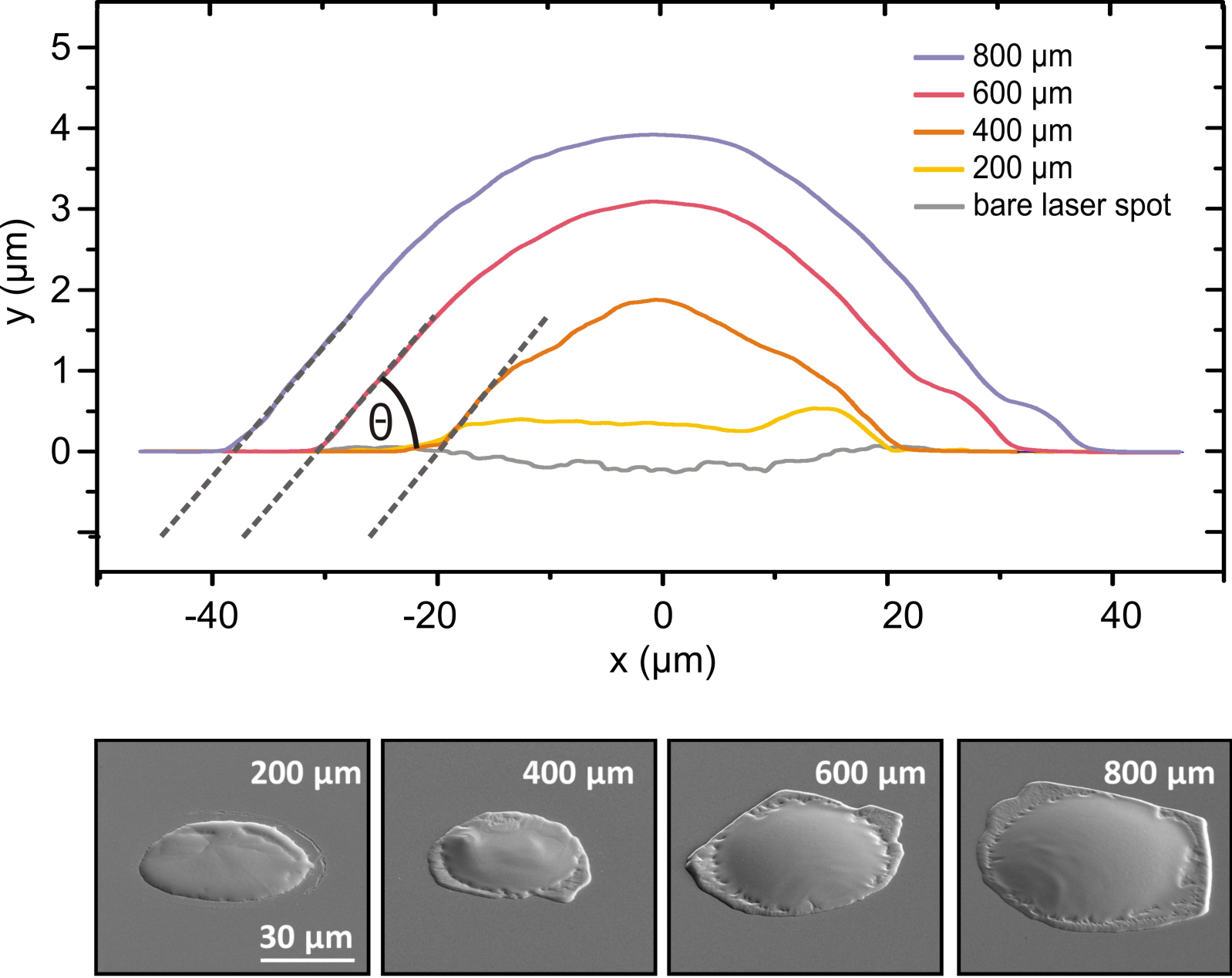


\section{Figure Captions}

Figure 1: Scheme of random vs. ordered indium island growth (adapted from Ref [6]). Left: Indium island preparation on non-patterned substrate (molybdenum film on glass). Right: Site-controlled arrangement of indium islands by fs laser patterning the glass substrate prior to deposition of molybdenum. Top: Scheme of individual processing steps. Bottom: optical micrographs at the end of the two different processing chains.

Figure 2: Influence of substrate temperature $(T)$ and deposition rate $(r)$ on the morphology of indium islands. SEM images were recorded at $52^{\circ}$ tilt with respect to the surface normal. Note the different magnifications of the images. Bottom: AFM [(a),(b) and profilometric (c),(d),(e)] cross sections plotted with equal scales.

Figure 3: Indium islands grown in $5 \times 5$ arrays with different spacings $(200 \mu \mathrm{m}-800 \mu \mathrm{m})$ between the individual laser ablation spots. Left: collages of SEM images of all islands in the arrays (View tilted by $52^{\circ}$ with respect to surface normal. Note the different scale bars). Right: Grayscale coding of the corresponding island volumes as derived from profilometric measurements.

Figure 4: Influence of the array spacing on the indium island contact angles and morphologies. Top: Profilometric scans across center of islands taken in arrays of different spacings (note the different scaling of $x$ and $y$ axes). The profile of the laser ablations spot (gray line) without deposition of indium is shown for comparison. The dashed lines indicate the contact angle $\left(\theta \sim 10^{\circ}\right)$ formed locally between the indium and the molybdenum coated substrate. Bottom: SEM images of the corresponding islands (view tilted by $52^{\circ}$ with respect to surface normal). 